Harry I. Miller, Department of Mathematics, Bilkent University, Ankara, Turkey - on leave from the University of Tennessee - Chattanooga. e-mail: hmiller@cecasun.utc.edu

Henry L. Wyzinski, Department of Mathematics, Indiana University N.W., Gary, IN 46408-1197. e-mail: hwyzin@iunhaw1.iun.indiana.edu

\title{
ON OPENNESS OF DENSITY POINTS UNDER MAPPINGS
}

\begin{abstract}
Basu and Ganguly recently proved a theorem connected to the classical theorem of Steinhaus which states that $A-B$ has nonempty interior if $A$ and $B$ are Lebesgue measurable subsets of the real line, each having positive measure. The Basu and Ganguly paper deals with a particular 2-place function, namely $f(x, y)=x / y$. There is nothing special about ratios. We will extend their results to functions satisfying simple conditions on their partial derivatives. An $n$ dimensional analogue is also presented.
\end{abstract}

\section{Introduction}

In a recent article [2] Basu and Ganguly proved that if $A_{1}, A_{2} \subseteq \mathbb{R}$ (the real line) are Lebesgue measurable and each has positive Lebesgue measure, then $R\left[A_{1}^{*}: A_{2}^{*}\right]$ is an open set, where $A_{i}^{*}=\left\{x \in \mathbb{R} \backslash\{0\}: A_{i}\right.$ has density 1 at $\left.x\right\}$ and $R\left[A_{1}^{*}: A_{2}^{*}\right]$ denotes the collection of all numbers $\frac{x}{y}$ and $\frac{y}{x}$ where $x \in$ $A_{1}^{*}, y \in A_{2}^{*}$. This result is a contribution to the collection of analogues and extensions of a theorem of Steinhaus [5], that goes back to 1920. The purpose of this note is to show that there is nothing special about ratios and that analogous statements in $\mathbb{R}$ and $\mathbb{R}^{n}$ hold for 2-place functions satisfying simple conditions on their partial derivatives. That the Steinhaus theorem can be extended using general functions is not a new idea - for example see [1], [3] and [4].

Key Words: Steinhaus theorem, density points

Mathematical Reviews subject classification: 28A05

Received by the editors May 12, 1998 


\section{Results}

We first consider the $n=1$ dimensional extension of the result of Basu and Ganguly mentioned in the introduction.

Theorem 1. Suppose that $f: \mathbb{R} \times \mathbb{R} \rightarrow \mathbb{R}, A$ and $B$ are Lebesgue measurable subsets of $\mathbb{R}$ and $x_{0} \in \widetilde{A}, y_{0} \in \widetilde{B}$ where $\widetilde{A}$ and $\widetilde{B}$ are the density points of $A$ and $B$, respectively. Assume further that $f_{x}, f_{y}$ (partial derivatives) exist and are continuous in a neighborhood of $\left(x_{0}, y_{0}\right)$ and that $f_{x}\left(x_{0}, y_{0}\right) \neq 0$ and $f_{y}\left(x_{0}, y_{0}\right) \neq 0$. Then $t_{0}:=f\left(x_{0}, y_{0}\right)$ is an interior point of $f(\widetilde{A}, \widetilde{B}):=$ $\{f(a, b): a \in \widetilde{A}, b \in \widetilde{B}\}$.

Proof. Given $\delta>0$, there exist $0<\delta_{1}, \delta_{2}, \delta_{3}<\delta$ such that for each $c \in$ $N_{3}:=\left(t_{0}-\delta_{3}, t_{0}+\delta_{3}\right)$ and each $x \in N_{1}:=\left(x_{0}-\delta_{1}, x_{0}+\delta_{1}\right)$ there exists a unique $y \in N_{2}:=\left(y_{0}-\delta_{2}, y_{0}+\delta_{2}\right)$ such that $f(x, y)=c$ and $f_{x}, f_{y}$ exist and are continuous and non-zero on $N_{1} \times N_{2}$. For each $c \in N_{3}$, let $g_{c}: N_{1} \rightarrow N_{2}$ be defined as above, i.e., $g_{c}(x)=y$ and $f\left(x, g_{c}(x)\right)=f(x, y)=c$. For each $c \in N_{3}$, $g_{c}^{\prime}(x)=\frac{-f_{x}\left(x, g_{c}(x)\right)}{f_{y}\left(x, g_{c}(x)\right)}$ for every $x \in N_{1}$. Set $M:=\frac{-f_{x}\left(x_{0}, g_{t_{0}}\left(x_{0}\right)\right)}{f_{y}\left(x_{0}, g_{t_{0}}\left(x_{0}\right)\right)}=\frac{-f_{x}\left(x_{0}, y_{0}\right)}{f_{y}\left(x_{0}, y_{0}\right)}$.

There exists a $d_{1}, 0<d_{1}<\delta_{1}$, such that $\frac{|M|}{2} d_{1}<\delta_{2}$ and $g_{c}^{\prime}(x)=M+$ $\varepsilon(x, c)$, where $|\varepsilon(x, c)|<\frac{|M|}{10}$ for all $c \in N_{3}$ satisfying $g_{c}\left(x_{0}\right) \in U_{3}:=\left(y_{0}-\right.$ $\left.\frac{|M|}{2} d_{1}, y_{0}+\frac{|M|}{2} d_{1}\right)$ and for all $x \in U_{1}:=\left(x_{0}-d_{1}, x_{0}+d_{1}\right)$ (notice $t_{0}$ is an interior point of these $c$ 's $)$ and such that $\frac{m\left(\widetilde{A} \cap U_{1}\right)}{2 d_{1}}>0.95$ and $\frac{m\left(\widetilde{B} \cap U_{2}\right)}{4|M| d_{1}}>0.95$ where $U_{2}:=\left(y_{0}-2|M| d_{1}, y_{0}+2|M| d_{1}\right)$.

For the c's mentioned above $g_{c}\left(\widetilde{A} \cap U_{1}\right)$ is a measurable set with measure greater than $\left(\frac{9|M|}{10}\right)\left(2 d_{1}\right)(0.95)$ and is contained in $U_{2}$ since $\frac{|M|}{2} d_{1}+\frac{11|M|}{10} d_{1}<$ $2|M| d_{1}$. This implies $g_{c}\left(\widetilde{A} \cap U_{1}\right) \cap\left(\widetilde{B} \cap U_{2}\right) \neq \emptyset$. Therefore, there exists an $x_{c} \in \widetilde{A} \cap U_{1}$ such that $g_{c}\left(x_{c}\right) \in \widetilde{B} \cap U_{2}$, or $f\left(x_{c}, g\left(x_{c}\left(x_{c}\right)\right)=c\right.$ with $x_{c} \in \widetilde{A}$, $g_{c}\left(x_{c}\right) \in \widetilde{B}$ for each $c \in U_{3}^{\prime}:=\left\{c \in N_{3}: g_{c}\left(x_{0}\right) \in U_{3}\right\}$ and $t_{0}$ is an interior point of $U_{3}^{\prime}$.

We now proceed to the $\mathrm{n}$ dimensional extension of the Basu, Ganguly result.

Theorem 2. Suppose that $f: \mathbb{R}^{n} \times \mathbb{R}^{n} \rightarrow \mathbb{R}^{n}$, A and $B$ are Lebesgue measurable subsets of $\mathbb{R}^{n}$ and $x_{0} \in \widetilde{A}, y_{0} \in \widetilde{B}$ where $\widetilde{A}$ and $\widetilde{B}$ are the density points of $A$ and $B$ respectively. Assume further that:

$(\alpha)$ The $2 n^{2}$ partial derivatives ( $n$ functions and $2 n$ variables) exist and are continuous in some neighborhood of $\left(x_{0}, y_{0}\right)$. 


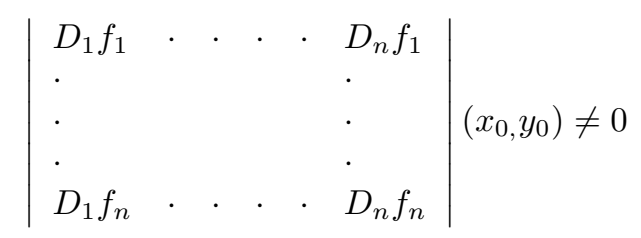

and

$$
\left|\begin{array}{llllll}
D_{n+1} f_{1} & \cdot & \cdot & \cdot & \cdot & D_{2 n} f_{1} \\
\cdot & & & & \cdot \\
\cdot & & & & \cdot \\
\cdot & & \cdot \\
D_{n+1} f_{1} & \cdot & \cdot & \cdot & \cdot & D_{2 n} f_{n}
\end{array}\right|\left(x_{0}, y_{0}\right) \neq 0
$$

where $f=\left(f_{1}, f_{2}, \ldots, f_{n}\right)$.

Then $t_{0}:=f\left(x_{0}, y_{0}\right)$ is an interior point of $f(\widetilde{A}, \widetilde{B}):=\{f(a, b): a \in \widetilde{A}, b \in$ $\widetilde{B}\}$.

Proof. $f$ can be viewed as an $n \times 1$ column matrix. The $n \times n$ matrices $\frac{\partial f}{\partial x}=\left[\frac{\partial f}{\partial x_{1}} \frac{\partial f}{\partial x_{2}} \cdots \frac{\partial f}{\partial x_{n}}\right]$ and $\frac{\partial f}{\partial y}=\left[\frac{\partial f}{\partial y_{1}} \frac{\partial f}{\partial y_{2}} \cdots \frac{\partial f}{\partial y_{n}}\right]$ are both invertible at $\left(x_{0}, y_{0}\right)$ by the hypotheses of this theorem. By the implicit function theorem, there is a continuously differentiable function $g(x, t)$ defined for $x$ near $x_{0}$ and $t$ near $t_{0}=f\left(x_{0}, y_{0}\right)$ such that $f(x, g(x, t))=t$. By implicit differentiation, we have $\frac{\partial g}{\partial x}=-\left[\frac{\partial f}{\partial y}\right]\left[\frac{\partial f}{\partial x}\right]$. Therefore $\frac{\partial g}{\partial x}$ is invertible at the point $\left(x_{0}, t_{0}\right)$. Hence, given $\delta>0$, there exist $\delta_{1}, \delta_{2}, \delta_{3}$, with $0<\delta_{1}, \delta_{2}, \delta_{3}<\delta$ such that for each $c \in N_{3}:=N\left(t_{0}, \delta_{3}\right)$, the open ball in $\mathbb{R}^{n}$ with center $t_{0}$ and radius $\delta_{3}$, and each $x \in N_{1}:=N\left(x_{0}, \delta_{1}\right)$, there exists a unique $y \in N_{2}:=N\left(y_{0}, \delta_{2}\right)$ such that $f(x, y)=c$. For each $c \in N_{3}$, let $g_{c}: N_{1} \rightarrow N_{2}$ be defined as above, i.e., $g_{c}(x)=y$ with $f\left(x, g_{c}(x)\right)=f(x, y)=c$.

Set $J_{g_{t_{0}}}\left(x_{0}\right)=\operatorname{det}\left(\frac{\partial g_{t_{0}}}{\partial x}\right)\left(x_{0}\right)=M_{1}$, which, by the above, is not zero.

By the assumption of the continuity of the $2 n^{2}$ partial derivatives there exists a $d_{1}, \quad 0<d_{1}<\delta$, such that $\frac{\left|M_{1}\right|}{2} d_{1}<\delta_{2}$ and $J_{g_{c}}(x)=M_{1}+\varepsilon(x, c)$ where $|\varepsilon(x, c)|<\frac{\left|M_{1}\right|}{10}$ for all $x \in U_{1}:=N\left(x_{0}, d_{1}\right)$ and for all $c \in C:=\{c \in$ $\left.N_{3} ; g_{c}\left(x_{0}\right) \in U_{3}:=N\left(y_{0}, \frac{\left|M_{1}\right|}{2} d_{1}\right)\right\}$ and $\left\|g_{c}(x)-g_{c}\left(x_{0}\right)\right\|<M_{2}\left\|x-x_{0}\right\|$ for every $x \in U_{1}$ and every $c \in C$ and $\frac{m\left(\widetilde{A} \cap U_{1}\right)}{m\left(U_{1}\right)}>\frac{1}{q}$ and $\frac{m\left(\widetilde{B} \cap U_{2}\right)}{m\left(U_{2}\right)}>\frac{1}{q}$ where $U_{2}:=N\left(y_{0}, M_{2} d_{1}+\frac{\left|M_{1}\right|}{2} d_{1}\right)$ and where $q=\left[\frac{9}{10}\left|M_{1}\right| \frac{m\left(U_{1}\right)}{m\left(U_{2}\right)}+1\right]$. 
Notice that $t_{0}=f\left(x_{0}, y_{0}\right)$ is an interior point of $C$. By the definitions of $U_{1}, U_{2}, U_{3}$ and $C, g_{c}\left(\widetilde{A} \cap U_{1}\right) \subseteq U_{2}$ for every $c \in C$ and $m\left(g_{c}\left(\widetilde{A} \cap U_{1}\right)\right)=$ $\int_{\widetilde{A} \cap U_{1}}\left|J_{g_{c}}(x)\right| d x>\frac{9}{10}\left|M_{1}\right| m\left(\widetilde{A} \cap U_{1}\right)>\frac{9}{10}\left|M_{1}\right| \frac{1}{q} m\left(U_{1}\right)$ for each $c \in C$.

Furthermore $\frac{9}{10}\left|M_{1}\right| \frac{1}{q} m\left(U_{1}\right)+\frac{1}{q} m\left(U_{2}\right)=\frac{1}{q}\left[\frac{9}{10}\left|M_{1}\right| \frac{m\left(U_{1}\right)}{m\left(U_{2}\right)}+1\right] m\left(U_{2}\right)=$ $m\left(U_{2}\right)$ and therefore $g_{c}\left(\widetilde{A} \cap U_{1}\right) \cap\left(\widetilde{B} \cap U_{2}\right) \neq \emptyset$ for every $c \in C$. Hence, for each $c \in C$, there exist $a_{c} \in \widetilde{A}$ and $b_{c} \in \widetilde{B}$ with $g_{c}\left(a_{c}\right)=b_{c}$ or $f\left(a_{c}, b_{c}\right)=c$. Therefore, $t_{0}$ is an interior point of $\{f(x, y): x \in \widetilde{A}, y \in \widetilde{B}\}$.

\section{References}

[1] P. Erdős and J. C. Oxtoby, Partition of the Plane into Sets Having Positive Measure in Every Non-null Measurable Product Set, Trans. Amer. Math. Soc. 79(1955), 91-102.

[2] D. K. Ganguly and S. Basu, On Some Properties of Sets with Positive Measure, Czech. Math. J. 44(119) 1994, 405-411.

[3] H. I. Miller, Generalization of a Classical Theorem of Measure Theory, Radovi XLV, Akademija Nauka i Umjetnosti BiH, knjiga 12, Sarajevo 1973, 45-48.

[4] W. Sander, Verallgemeinerungen eines satzes von H. Steinhaus, Manuscripta Mathematica, 18(1976), 25-42.

[5] H. Steinhaus, Sur les distances des points des ensembles de mesure, Fund. Math. 1(1920), 93-104. 